\title{
Autophagic or necrotic cell death triggered by distinct motifs of the differentiation factor DIF-1
}

\author{
MF Luciani ${ }^{1,2,3}$, Y Kubohara ${ }^{4}$, H Kikuchi $^{5}$, Y Oshima ${ }^{5}$ and P Golstein ${ }^{\star, 1,2,3}$
}

\begin{abstract}
Autophagic or necrotic cell death (ACD and NCD, respectively), studied in the model organism Dictyostelium which offers unique advantages, require triggering by the same differentiation-inducing factor DIF-1. To initiate these two types of cell death, does DIF-1 act through only one or through two distinct recognition structures? Such distinct structures may recognize distinct motifs of DIF-1. To test this albeit indirectly, DIF-1 was modified at one or two of several positions, and the corresponding derivatives were tested for their abilities to induce ACD or NCD. The results strongly indicated that distinct biochemical motifs of DIF-1 were required to trigger ACD or NCD, and that these motifs were separately recognized at the onset of ACD or NCD. In addition, both ACD and NCD were induced more efficiently by DIF-1 than by either its precursors or its immediate catabolite. These results showed an unexpected relation between a differentiation factor, the cellular structures that recognize it, the cell death types it can trigger and the metabolic state of the cell. The latter seems to guide the choice of the signaling pathway to cell death, which in turn imposes the cell death type and the recognition pattern of the differentiation factor.
\end{abstract}

Cell Death and Differentiation (2009) 16, 564-570; doi:10.1038/cdd.2008.177; published online 12 December 2008

In animal cells, apoptosis has long been considered the major if not the exclusive type of cell death. However, other cell death types can occur, such as autophagic or necrotic cell death (ACD or NCD, respectively). Although the pathophysiological importance of ACD and NCD is increasingly appreciated, ${ }^{1-5}$ they are still incompletely defined.

The cellular slime mold Dictyostelium discoideum is an excellent eukaryote model organism for both cell and developmental biology. Its cells grow as single amebae in rich medium, but when starved start a developmental program of morphogenesis and gather to form a fruiting body comprising a multi-cellular stalk. In this stalk, cells are dying or dead, thus providing an example of developmental cell death. The latter can be mimicked and studied under in vitro monolayer conditions. ${ }^{6}$

Three main advantages make Dictyostelium an exceptionally favorable model to study the induction of nonapoptotic cell death. First, its small, sequenced and haploid genome $\mathrm{e}^{7-9}$ facilitates mutagenesis and other genetic approaches. Second, the Dictyostelium genome includes no genes of the two main molecular families (caspases and bcl-2 family) ensuring apoptosis in animal cells, except one paracaspase that we found not to be involved in cell death. ${ }^{10,11}$ Thus, in Dictyostelium cells there is no caspase/bcl-2-dependent apoptosis machinery that could mediate apoptosis or interfere with nonapoptotic cell death.

Third, and most importantly, induction of cell death in Dictyostelium under in vitro monolayer conditions requires two distinct stimuli (Figure 1a), first starvation (and adenosine $3^{\prime}-5^{\prime}$-cyclic monophosphate (cAMP), which will not be studied nor discussed separately from starvation here), then stimulation by differentiation-inducing factor-1 (DIF-1), a major DIF in Dictyostelium. ${ }^{6,12-15}$ DIF-1 is a dichlorinated alkyl phenone that is required for induction of cell death in vitro ${ }^{6,16,17}$ (Figure 1b). In some Dictyostelium strains such as HMX44A (see below), DIF-1 is not made in sufficient amounts by starving cells in vitro, which thus require addition of exogenous DIF-1 to die.

The same two signals, starvation and DIF-1, were required for induction of distinct types of cell death, however in different cellular circumstances. When wild-type Dictyostelium cells were subjected to starvation and DIF-1, they underwent ACD, with emergence of paddle cells and IP3R-dependent and transcription factor-dependent vacuolization and death. ${ }^{16-21}$ When cells mutated for the atg1 autophagy gene were similarly treated, they underwent NCD instead, with mitochondrial uncoupling causing perinuclear clustering, then lysosomal and plasma membrane alterations (22,23; Giusti et al., in preparation; Figure 1a). Remarkably, these two different types of death both required induction by DIF-1, in cells subjected to either starvation alone (wt cells, induced to undergo ACD), or starvation aggravated by inhibition of autophagy ( $\operatorname{atg} 1^{-}$cells, induced to undergo NCD). Thus, the 'starvation context' of the cell modified the outcome of signaling by DIF-1.

Autophagic cell death or NCD following addition of DIF-1 could be due to recognition of DIF-1 by the same structure $\left(S_{0}\right.$ in Figure 1a), leading through a common initial stretch, then distinct downstream pathways to either mitochondrial lesions and NCD, or to other lesions and ACD. Alternatively,

${ }^{1}$ Centre d'Immunologie de Marseille-Luminy, Aix-Marseille Université, Marseille F-13288, France; ${ }^{2}$ INSERM U631, Marseille F-13288, France; ${ }^{3}$ CNRS UMR6102, Faculté des Sciences de Luminy, Marseille F-13288, France; ${ }^{4}$ Institute for Molecular \& Cellular Regulation, Gunma University, Maebashi 371-8512, Japan and ${ }^{5}$ Laboratory of Natural Product Chemistry, Graduate School of Pharmaceutical Sciences, Tohoku University, Sendai 980-8578, Japan

*Corresponding author: P Golstein, Centre d'Immunologie de Marseille-Luminy, Case 906, Faculté des Sciences de Luminy, 13288 Marseille cedex 9 , France.

Tel: + 334912694 68; Fax: + 334912694 30; E-mail: golstein@ ciml.univ-mrs.fr

Keywords: autophagic cell death; necrotic cell death; Dictyostelium; DIF-1

Abbreviations: ACD, autophagic cell death; DIF-1, differentiation-inducing factor-1; NCD, necrotic cell death; ROS, reactive oxygen species

Received 23.9.08; revised 06.11.08; accepted 07.11.08; Edited by P Mehlen; published online 12.12 .08 
a
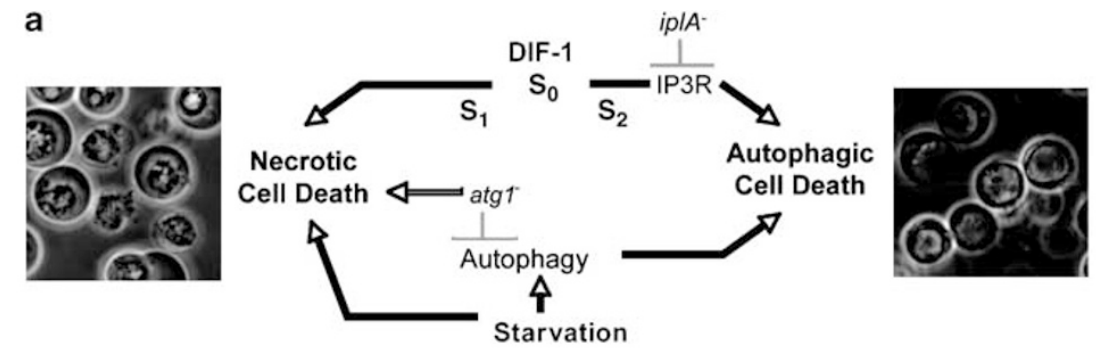

b<smiles>CCCCCC(=O)c1c(O)c(Cl)c(OC)c(Cl)c1O</smiles>

DIF derivatives: radical at the indicated position

\begin{tabular}{llrllll} 
Name & R1 & R2 & R4 & R4 & R5 & R6 \\
\hline DIF-1 & CO- $(\mathrm{CH} 2)_{4}-\mathrm{CH}_{3} \mathrm{OH}$ & $\mathrm{Cl}$ & $\mathrm{OCH} 3$ & $\mathrm{Cl}$ & $\mathrm{OH}$ \\
THPH & & $\mathrm{H}$ & $\mathrm{OH}$ & $\mathrm{H}$ & \\
DIF-3 & & & $\mathrm{H}$ & $\mathrm{OH}$ & & \\
107 & & $\mathrm{CO}-(\mathrm{CH} 2)_{6}-\mathrm{CH}_{3}$ & $\mathrm{H}$ & & & \\
114 & $\mathrm{CO}-(\mathrm{CH})_{6}-\mathrm{CH}_{3}$ & & & &
\end{tabular}

Figure 1 Dictyostelium cell death types, DIF-1 that induce them, and derivatives thereof. (a) The differentiation factor DIF-1 can induce autophagic vacuolar cell death (ACD, right) or necrotic cell death (NCD, left) provided the cells are first subjected to starvation. The choice between DIF-1-induced cell death types is a function of the state of these starved cells. In wild-type cells, ACD occurs, ${ }^{16-21}$ and this requires the IP3R probably to ensure Ca2 ${ }^{+}$flux from the ER to the cytosol. ${ }^{18}$ When autophagy cannot take place because of an atg $1^{-}$mutation, NCD occurs. ${ }^{22,23}$ The DIF-1-induced pathways to NCD or ACD could have a common $\left(\mathrm{S}_{0}\right)$ or distinct $\left(\mathrm{S}_{1}, \mathrm{~S}_{2}\right)$ DIF-1-recognizing starting points. (b) The structure of DIF- $1^{12}$ with position numbering. (c) Some derivatives thereof, selected on the basis of distinct behavior for induction of ACD or NCD

recognition of DIF could be ensured by distinct structures $\left(S_{1}\right.$ and $S_{2}$ in Figure 1a), and the corresponding distinct downstream events would lead to NCD or ACD, respectively. In this case, these death pathways would be distinct from the start. If the DIF-1-recognizing structures are different, what they recognize on DIF-1 might be different.

In this study, we tested derivatives, differing from DIF-1 by substitutions at one or two positions, for their ability to trigger ACD or NCD. We found that distinct motifs of DIF-1 were required to trigger these cell death types. Thus, the same differentiation factor DIF-1 was recognized differently to effect distinct cell death pathways, as a function of the starvation context of the cell.

\section{Results}

In preliminary experiments, DIF-1 (Figure 1b) and 21 derivatives thereof ${ }^{24}$ were tested for their ability to induce ACD or NCD on Dictyostelium HMX44A and the corresponding atg $1^{-}$HMX44A.atg1-1 mutant cells. ${ }^{22}$ All figures show only results obtained in the HMX44A background, whereas the main results in this report have also been obtained in the DH1 background (not shown). A few of the 21 DIF-1 derivatives, listed in Figure 1c, were found especially informative and were studied in more detail.

Differential induction of autophagic and necrotic cell death. Autophagic cell death was tested on HMX44A cells, checked by microscopy in terms of cell vacuolization and quantified through regrowth tests. ${ }^{16}$ NCD was tested on HMX44A.atg $1^{-}$cells, checked by microscopy in terms of appearance of perinuclear clustering and cell debris and quantified through scatter changes by FACS. ${ }^{23}$ There was consistent agreement between microscopy and quantitative tests.
In a representative experiment (Figure 2), the ability of DIF-1 to stimulate either ACD (shown as vacuolization (Figure 2a) and nonregrowth (Figure 2b)) or NCD (shown as perinuclear clustering and debris (Figure 2c) and membrane rupture (Figure $2 \mathrm{~d}$ )) was plotted as a function of its molar concentration and was taken as a reference (red lines). Compared to this reference, derivative 107 showed about the same ability to stimulate ACD (Figure $2 a$ and b; full green line), but far less ability to stimulate NCD (Figure $2 \mathrm{c}$ and d; dotted green line). This was found in each of more than 15 experiments (not shown). Out of these, 8 used high concentrations of 107 , leading to the conclusion that the same NCD-inducing efficiency required concentrations of 107 one to two orders of magnitude higher than those of DIF-1 (Figure $3 \mathrm{~b}$ ), even if NCD was assessed $5 \mathrm{~h}$ after addition of DIF-1 (Figure 3b). In parallel, in these experiments the ACDinducing efficiency was within the same order of magnitude for 107 and for DIF-1 (Figure 2b), although sometimes less marked for the former (Figures $3 c$ and $4 b$ ).

Compared to DIF-1, 107 was less able to induce NCD, not only in terms of plasma membrane rupture, but also in terms of mitochondrial perinuclear clustering (Figure 3a) and induction of $2^{\prime}, 7^{\prime}$-dichlorofluorescin diacetate (DC-FDA) fluorescence (an indicator of ROS production, here in mitochondria ${ }^{23}$ ) qualitatively (Figure 3a) and quantitatively (Figure $3 b$ ). Although these traits were checked here $30 \mathrm{~min}$ after addition of DIF-1, DC-FDA fluorescence has been shown to occur in this system as soon as $3 \mathrm{~min}$ after addition of DIF-1. ${ }^{23}$ As DC-FDA fluorescence is mitochondrial and reflects mitochondrial uncoupling causative of downstream NCD events, ${ }^{23}$ these results indicate that the lesser effect of 107 compared to that of DIF-1 is inflicted at or before mitochondrial uncoupling.

Interestingly, derivative 114 showed a behavior reciprocal to that of 107. Compared to DIF-1, 114 showed far less ability to stimulate ACD and about the same ability to stimulate NCD 


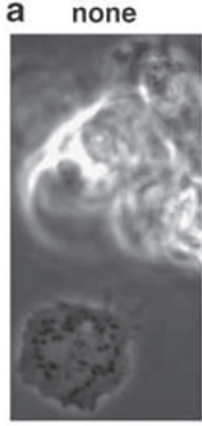

DIF-1

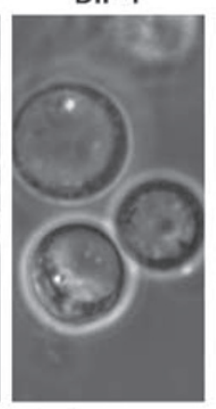

Autophagic cell death

C none

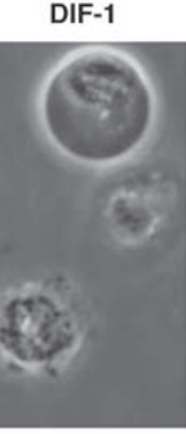

Necrotic cell death
114

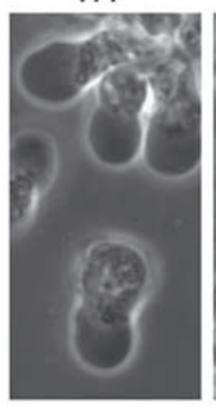

114

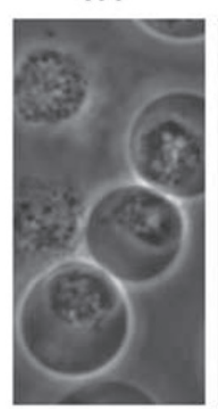

107

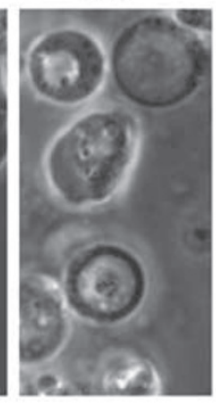

107

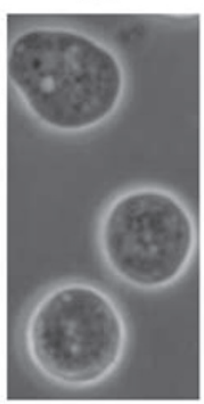

b

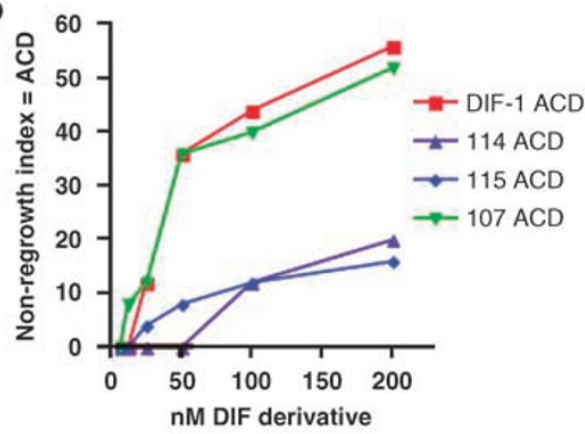

d

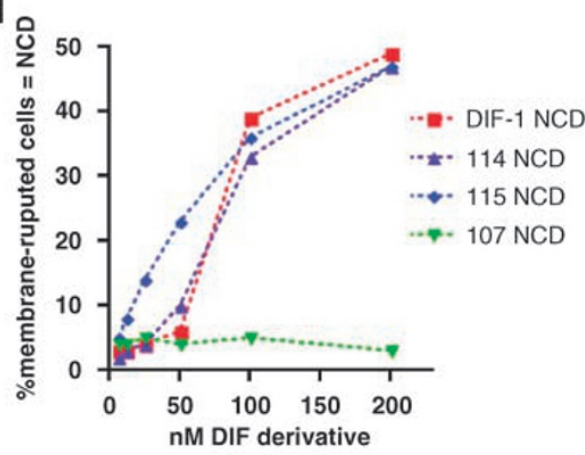

Figure 2 Preferential induction of either autophagic or necrotic cell death by derivatives 107, or 114 and 115. (a) ACD was tested on HMX44A cells. Under starvation only, the cells had a typical clumped or flat appearance (extreme left). Addition of $200 \mathrm{nM}$ of DIF-1 or of 107 led after $24 \mathrm{~h}$ to the usual vacuolar ACD, whereas addition of $200 \mathrm{nM}$ of 114 led to emergence and persistence of paddle cells with only little vacuolization. (b) Quantification of ACD showing as a function of concentration that 114 or 115 induce ACD less efficiently than DIF-1 or 107. (c) NCD was tested on HMX44A.atg1- cells. Under starvation only, the cells had a typical round appearance (extreme left). Addition of $200 \mathrm{nM}$ of DIF-1 or of 114 led after $1.5 \mathrm{~h}$ to the usual NCD with perinuclear clustering and debris, whereas addition of $200 \mathrm{nM}$ of 107 did not seem to alter the round cells. (d) Quantification of NCD showing as a function of concentration that 107 induces NCD less efficiently than DIF-1, 114 or 115

(Figure 2a-d, full and dotted purple lines, respectively) and to trigger DC-FDA fluorescence (not shown). At $200 \mathrm{nM}$, although DIF-1 consistently induced vacuolization as checked by microscopy and cell death as checked by regrowth tests, 114 induced neither in 8 out of 9 experiments. Also, although 114 did not induce vacuolization and cell death, it did induce paddle cells (Figure 2a), suggesting these are induced through a pathway distinct from that inducing vacuoles in agreement with previous mutagenesis results. ${ }^{18}$ Induction of paddle cells has not been investigated further because it could not be quantified easily. In all these experiments, 107 and 114 were tested on either wild type for ACD or atg $1^{-}$cells for NCD, but similar results were obtained when 107 and 114 were tested on wild-type cells under stringent conditions favoring either ACD or NCD (not shown). Also, the same reciprocal hierarchy of induction by 107 or 114 of ACD or NCD was found with $\mathrm{DH} 1$ and $\mathrm{DH} 1$. atg1 $^{-}$cells (not shown). Derivatives 107 and 114 were directly compared in four experiments, at a range of concentrations, for their ability to induce ACD or NCD on starved HMX44A or HMX44A.atg $1^{-}$cells, respectively. Statistical $t$-tests confirmed that in particular at a concentration of $100 \mathrm{nM}, 107$ was more efficient than 114 to induce ACD $(P$-value $=0.02)$, but less efficient than 114 to induce NCD $(P$-value $=0.008)$. Altogether, given modifications of DIF-1 differentially led to less induction of $A C D$ or less induction of NCD.
Differential lack of induction rather than inhibitory activity. Derivative 107 might lead to less NCD (and reciprocally 114 may lead to less $A C D$ ), either because it does not induce it, or because it actively inhibits it. Absence of induction or active inhibition would have different implications as to recognition of DIF-1 and its derivatives. We subjected cells to a mixture of 107 and 114 at equal concentrations (marked deviation from equal concentrations was impractical because of derivative effects at high concentrations). Derivative 114 did not inhibit ACD induction by 107 (Figure 3c), and derivative 107 did not inhibit NCD induction by 114 (Figure 3d). Thus, 107 and 114 may not actively inhibit NCD or ACD, but rather passively fail to induce NCD or ACD, respectively. Taken together, these and the results above indicate that 107 and 114 trigger less efficiently either NCD or ACD, respectively, through lesser recognition at the onset of one but not the other pathway.

Autophagic cell death-subefficient derivative 114 is distinct from DIF-1 at positions R1 and R3 (Figure $1 \mathrm{~b}$ and $\mathrm{c}$ ). Derivative 115 (similar to 114 at R1, and to DIF-1 at R3) behaved like 114 (Figure $2 \mathrm{~b}$ and $\mathrm{d}$ and not shown), which implies that it is mainly the modification at R1, which cripples 114. Also, NCD-subefficient derivative 107 is distinct from DIF-1 at position R4. Altogether, in the DIF-1 molecule R4 seems important for NCD induction, and R1 for ACD induction, showing that substitutions at given positions of this 
a

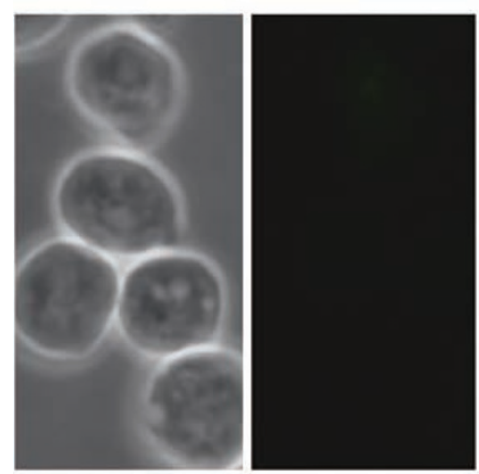

DIF-1

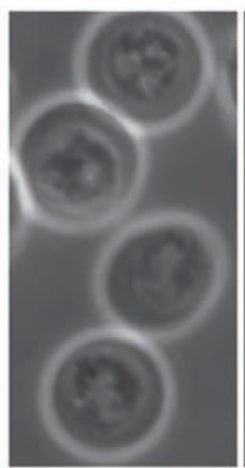

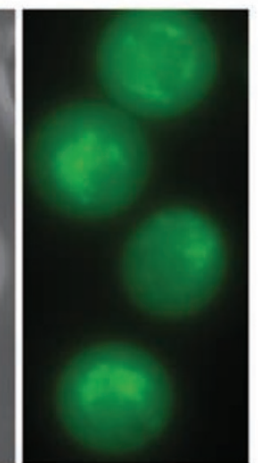

107
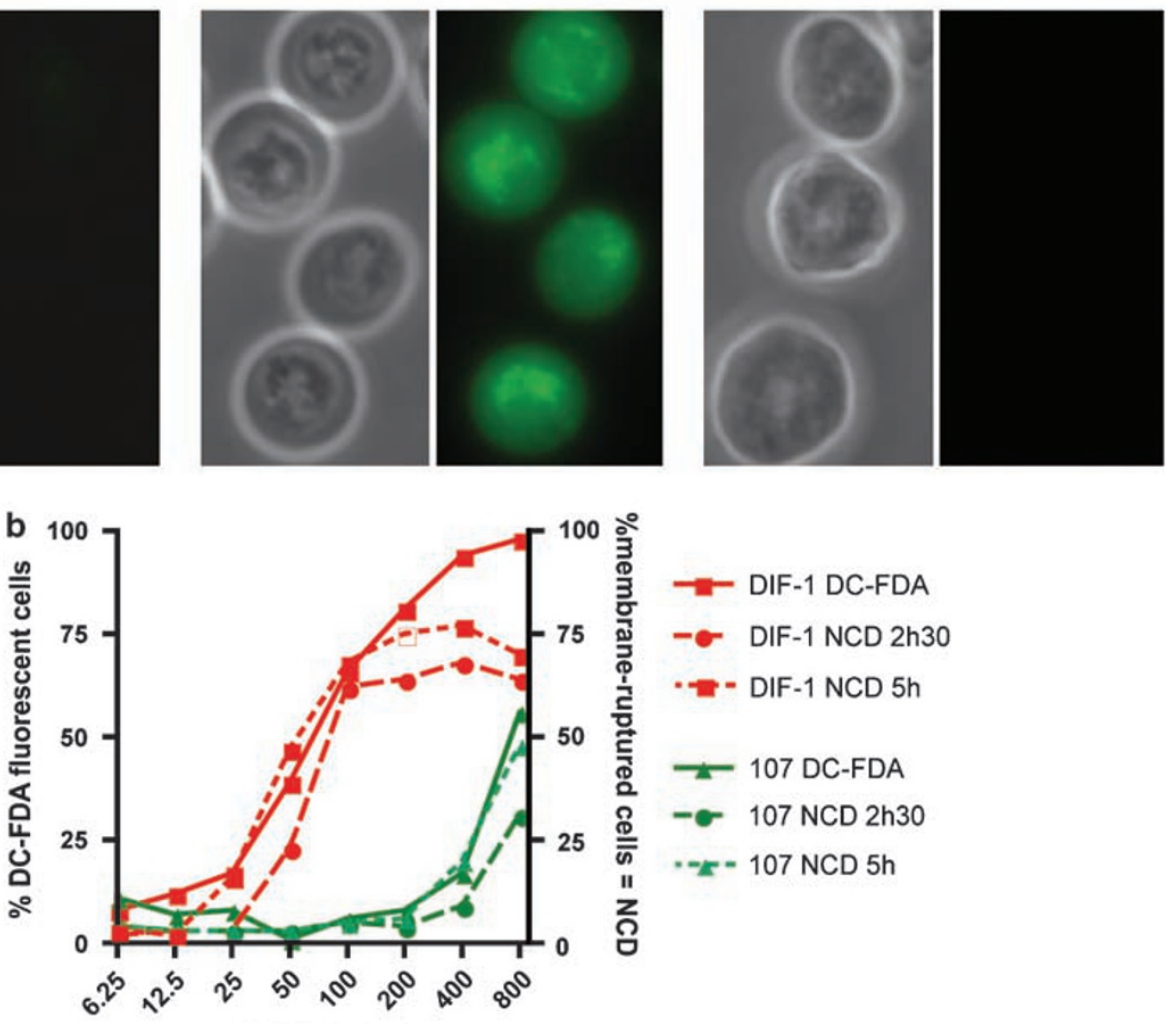

nM DIF derivative
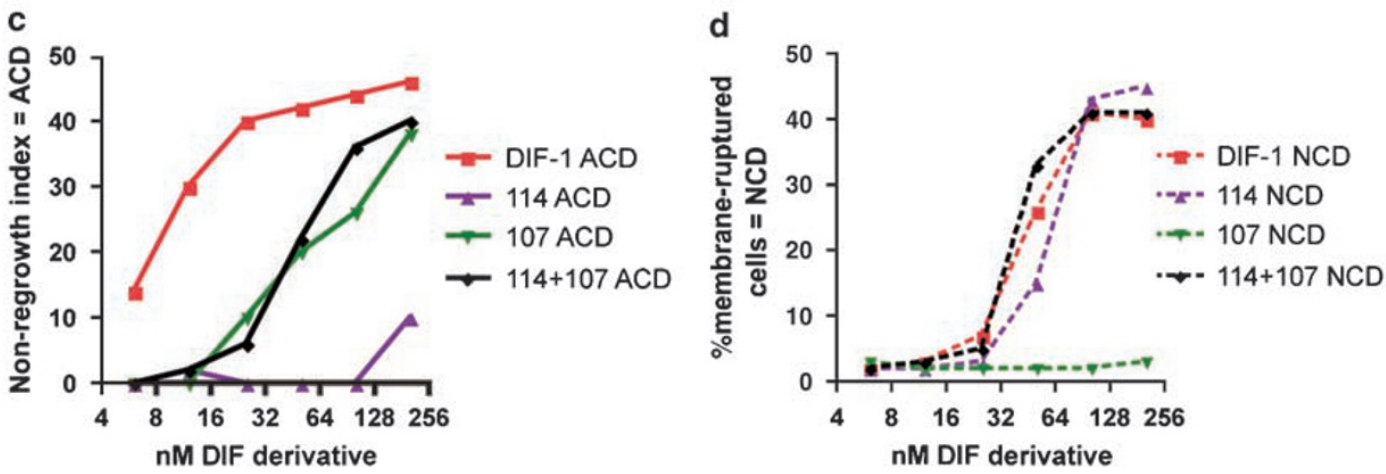

Figure 3 The difference between DIF-1 and 107 as to efficiency of induction of NCD can be detected at early steps thereof, and mixing 107 and 114 did not reveal any inhibitory activity of either on ACD or NCD. (a) Starved HMX44A.atg $1^{-}$cells were examined 30 min after addition of DIF-1 or 107 and 15 min after addition of DC-FDA. Left, control cells with no addition of DIF-1 or 107 were round and not fluorescent. Middle, addition of $100 \mathrm{nM}$ DIF-1 led to the usual perinuclear clustering and fluorescence of mitochondria. Right, addition of $100 \mathrm{nM} 107$ led to neither detectable clustering nor fluorescence. (b) In a separate experiment, quantification by FACS of plasma membrane rupture at 2.5 and $5 \mathrm{~h}$, and of DC-FDA fluorescence at $30 \mathrm{~min}$ after addition of a range of concentrations of either DIF-1 or 107. Compared to DIF-1, 107 was less able to induce NCD in terms of both plasma membrane-rupture and early DC-FDA fluorescence. (c) The mixture of 107 and 114 was not less efficient than 107 alone to induce ACD, and (d) was not less efficient than 114 alone to induce NCD, showing that neither 107 nor 114 had inhibitory activity on the type of cell death that they did not induce

small molecule matter for the triggering of one or the other cell death pathway.

Death-inducing activity of precursors or catabolites of DIF-1. Immediate precursors and catabolites of DIF-1 were identified by Kay and colleagues ${ }^{13,25,26}$ (Figure 4a). We investigated their death-inducing ability. THPH (Figure 1c) had little ACD- or NCD-inducing ability, whereas interestingly at the same concentrations it triggered paddle cell formation (not shown). The chloroperoxidase-mediated acquisition of two chlorine radicals led to gain of ACD (but not NCD)inducing ability (compare THPH and 107; Figure 4b). Further methyltransferase-mediated replacement of the hydroxyl by an $\mathrm{OCH} 3$ group at R4 led to DIF-1 and resulted in gain of NCD-inducing ability (compare 107 and DIF-1; Figure 2a). DIF-1 was thus able to induce both ACD and NCD. A dechlorinase led to loss of the chlorine at R3, with the resulting moderate (less than one order of magnitude) decrease in ability to induce both ACD and NCD (compare DIF-1 and DIF-3; Figure 4c). This suggests that, of the two 
<smiles>CCCCCC(=O)c1c(O)cc(O)c(O)c1O</smiles><smiles>CCCCCC(=O)c1c(O)c(Cl)c(OC)c(Cl)c1O</smiles>
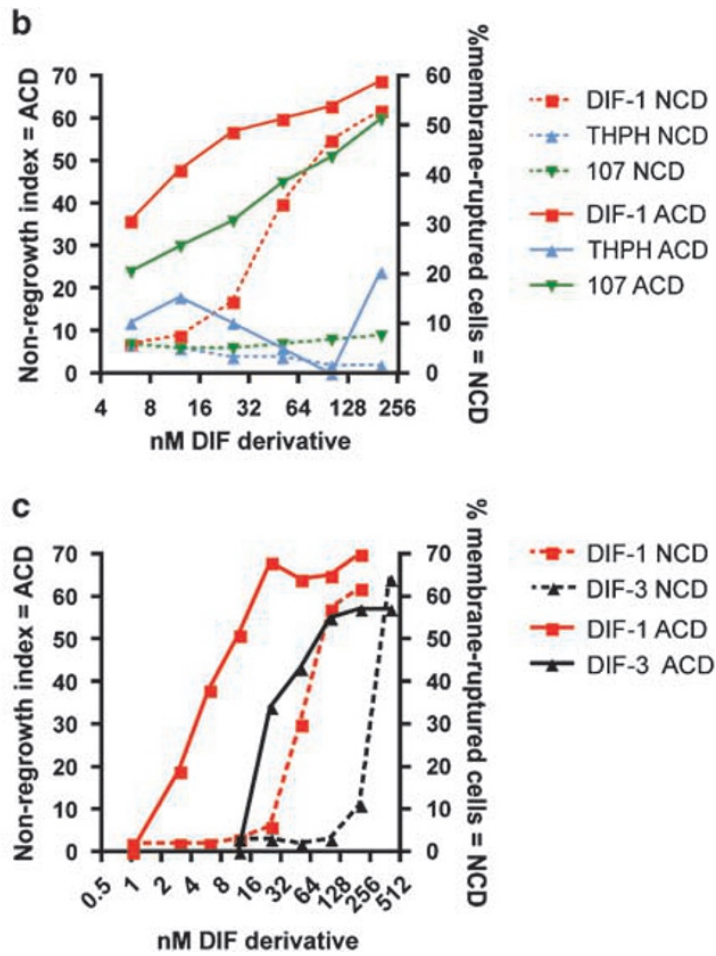

Figure 4 Efficiency of induction of ACD or NCD by natural precursors and catabolites of DIF-1. (a) The sequence of products and of corresponding enzymes on the DIF-1 pathway as previously established. ${ }^{13,25,26}$ (b) In the range of concentrations shown, THPH induces neither ACD nor NCD, 107 induces ACD not NCD, DIF-1 induces both. (c) DIF-3 induces both ACD and NCD but somewhat less efficiently than DIF-1

chlorine that conferred ACD-inducing ability to 107, the one at $\mathrm{R} 5$ mattered most.

Altogether (Figure 4), on the DIF-1 biosynthetic pathway from THPH to DIF-1, there was acquisition of ability to induce, first ACD, then also NCD. On the DIF-1 catalytic pathway from DIF-1 to DIF-3, both abilities somewhat decreased. DIF-1 thus seems to represent an optimum, leading one to wonder what could be the selective advantage of this optimization. In particular, that DIF-1 should be optimal not only for ACD induction, but also for NCD induction is an argument in favor of a physiological role also for NCD (or for a function related to NCD).

\section{Discussion}

This report shows that in starved HMX44A cells, DIF-1 recognized according to a given pattern triggered $A C D$; and that in starved HMX44A.atg1-1 cells, DIF-1 recognized according to another pattern triggered NCD. The atg $1^{-}$ autophagy gene mutation would aggravate the starving state of the cell. These results thus indicate the existence of a relation among a differentiation factor, the cellular structures that recognize it, the cell death types it can trigger and the metabolic state of the cell. In principle, differential effects of DIF-1 derivatives could be either because of distinct receptors recognizing different regions of DIF-1, or to different accessibility to the same receptor located in distinct subcellular structures. The latter explanation would require that DIF-1 can access say structures $A$ and $B, 107$ only $A$ and 114 only $B$. These reciprocal shifts in accessibility through limited modifications of a small molecule seem unlikely. Also, this explanation would require that the same receptor in different locations could trigger entirely different downstream cascades. Although this cannot be ruled out, we favor the other explanation, of distinct 'receptors' recognizing DIF-1 from different angles. 'Receptor' is taken here in a broad sense, stretching from a classical lock-and-key molecular fit to local physical conditions favoring charge or hydrophilicity.

As paddle cell formation is not easily quantifiable, we did not systematically investigate whether, within ACD, and between derivatives, there could be a dissociation between induction of vacuolization and death and induction of paddle cells. Still, it is remarkable that DIF-1 would induce vacuolization and death in the 10-100 nM range, whereas it would induce paddle cells already at much lower concentrations $(0.01-0.1 \mathrm{nM})$, and that $\mathrm{THPH}$ at given concentrations induced paddle cells but neither ACD nor NCD. These results (not shown in detail), and the fact that paddle cell induction by DIF-1 could be genetically distinguished from ACD vacuolization and death $^{18}$, are in line with different pathways to paddle cell formation and to vacuolization, but they do not allow firm conclusions as to whether there is only one or distinct receptors in these cases. Altogether, these studies indicated the existence in/on Dictyostelium cells of at least two (leading to NCD or ACD), and perhaps three (also leading to paddle cells) distinct DIF-1-recognizing 'receptors'.

To explain how a given type of cell death seems linked to a given pattern of reactivity to DIF-1 derivatives as a function of the state of the cell, we propose the following simple hypothesis (Figure 5). Irrespective of the metabolic state of the cell, DIF-1 would bind to several 'receptors', each able to trigger a distinct type of cell death through its corresponding 


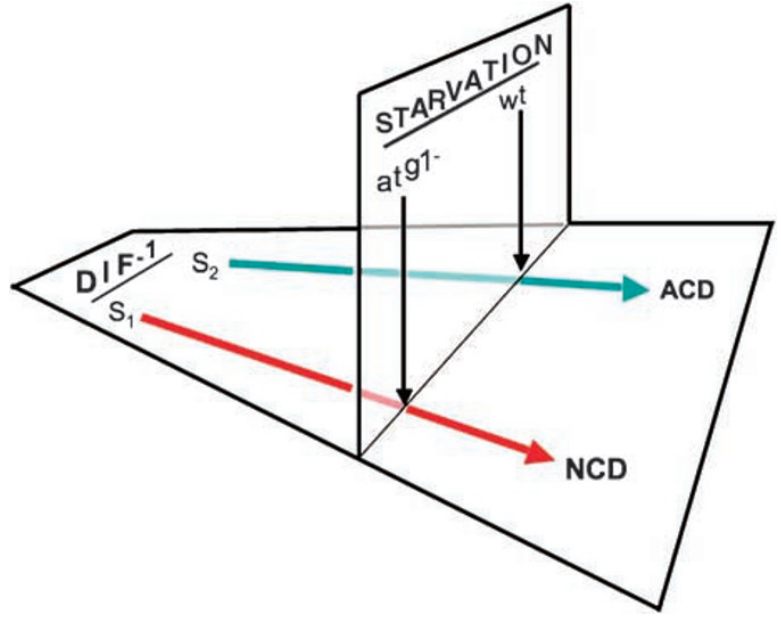

Figure 5 A working hypothesis to explain the relationship between cell context, type of cell death and distinct DIF-1 recognition patterns. Distinct DIF-1 recognition structures $\left(\mathrm{S}_{1}, \mathrm{~S}_{2}\right)$ may exist on/in Dictyostelium cells. Exogenously added DIF-1 would engage all of these structures. One of the corresponding downstream pathways (colored arrows) leading to distinct cell death types would become sensitive to triggering by DIF-1 only as a function of a given state of the cell (black arrows). This sensitization of one of the pathways, as a function of the cellular context, would thus lead to one type of cell death, and would reveal the recognition pattern of only one of the DIF-1 recognition structures

downstream pathway. For NCD, given receptors (or physical conditions) would allow recognition/location of say DIF-1 and 114 but less so of 107 at the mitochondrial internal membrane, leading to uncoupling. Arguments for mitochondria as a direct target for DIF-1 at the onset of NCD are immediate uncoupling on addition of DIF-1 ${ }^{23}$ and uncoupling of isolated mitochondria on addition of DIF-1.27 For ACD, there could be recognition of both DIF-1 and 107 but less so of 114 at another site, perhaps at the plasma membrane, which would be consistent with a role for the IP3R ${ }^{18}$ downstream of this recognition. The metabolic state of the cell (starvation in wt or atg $1^{-}$cells) would favor the triggering of one of these pathways. Such prioritizing of a given pathway would lead, downstream, to a given type of cell death, and, upstream, to the apparent use of only the corresponding receptor, with its own pattern of reactivity to given DIF-1 derivatives. In short, the state of the cell would select a given pathway, connected to both a type of cell death and a pattern of recognition (Figure 5). An implication of this hypothesis is that a search for a given DIF-1 receptor should make use of well-defined test conditions including derivatives with a more restricted spectrum of induction than DIF-1 itself (for instance wt cells, induction by 107), to compensate for the possible 'noise' by other receptors for DIF-1.

Differentiation-inducing factor-1 (DIF-1) seems optimized for both ACD- and NCD-inducing abilities, suggesting that being able to undergo either type of cell death is a selective advantage for Dictyostelium. This also indicates that the ability of DIF-1 to be recognized in distinct ways, to lead to ACD or NCD, may be biologically significant.

We show in this report that a given differentiation factor can be recognized in distinct ways, leading to distinct consequences. Some published occurrences of relationships between ligands, receptors and effects are similarly distinct from mere one-to-one relationships. Thus, for instance TNFor ERBB ligand-superfamily members are able to induce multiple effects sometimes through distinct receptors. ${ }^{28,29}$ NGF family members can be recognized by distinct receptors and have distinct functions, ${ }^{30}$ and the same situation applies to netrin-1. ${ }^{31}$ Perhaps the main novel point brought about by this report is that for a given ligand, here a differentiation factor, the choice of both the operational receptors and the resulting effects may be coordinately guided by the metabolic state of the cell.

\section{Materials and Methods}

Materials. D. discoideum axenic strains HMX44A (see for derivation 17), HMX44A.atg1-1 (HMX44A mutated for the autophagy gene atg1, 22) were routinely grown at $22^{\circ} \mathrm{C}$ in $\mathrm{HL} 5$ medium ${ }^{32}$ as modified. ${ }^{16,18}$ Blasticidin $(10 \mu \mathrm{g} / \mathrm{ml})$ was added to the HMX44A.atg1-1 cultures. The HMX44A background was chosen because on starvation these cells produce very little endogenous DIF $-1,{ }^{33}$ the death pathways in this background have been studied in some detail ${ }^{16,17,22,23}$ and dying cells show features more clearcut than in most other strains; accordingly, only experiments in this background are shown. DH1 cells bearing the atg $1^{-}$mutations were similarly grown and tested (no shown). DIF-1, DIF-3 and their derivatives were synthesized as previously described. ${ }^{24}$

Inducing and revealing autophagic cell death. For induction of vacuolar death of HMX44A cells, ${ }^{16,17}$ exponentially growing vegetative cells were washed once in phosphate-buffered saline (Soerensen buffer; SB), and incubated in SB containing $3 \mathrm{mM}$ cAMP (Sigma) for $8 \mathrm{~h}$ at $22^{\circ} \mathrm{C}$ in Lab-Tek culture chambers (ref. 155380; Nalge Nunc) at a concentration of $3 \times 10^{5}$ cells $/ 1 \mathrm{ml} / \mathrm{chamber}$. Cells were then washed in $\mathrm{SB}$ and incubated at $22^{\circ} \mathrm{C}$ in either $\mathrm{SB}$ alone or $\mathrm{SB}$ containing the differentiation factor DIF-1 (DN1000; Affiniti Research Products, Exeter, UK) or derivatives thereof. After incubation for the indicated period of time, cells in the LabTek chambers were examined by phase contrast microscopy using an inverted Zeiss Axiovert 200 microscope usually with a $\times 100$ oil immersion objective. Pictures were taken using an Axiocam MRC digital camera connected to a PC equipped with Axiovision (Zeiss). Images were transferred to a Macintosh computer and treated with Graphic Converter. Figure assemblies were done using Illustrator.

Quantitation of cell death was through a regrowth test modified from Levraud et al. ${ }^{17} \mathrm{HMX} 44 \mathrm{~A}$ cells were incubated as indicated above in the presence or in the absence of DIF-1 or of derivatives thereof, for $24 \mathrm{~h}$ at $22^{\circ} \mathrm{C}$. To initiate regrowth of surviving cells, for each Lab-Tek chamber $0.5 \mathrm{ml}$ of SB was removed and $1 \mathrm{ml} \mathrm{HL}-5$ was added. Following 48 to $72 \mathrm{~h}$ of additional incubation at $22^{\circ} \mathrm{C}$, vegetative cells resulting from regrowth were counted. For each chamber, the number of cells was subtracted from the number of cells in control wells initially subjected to starvation only. The resulting figures reflected cell death induced by DIF-1 or derivatives. These figures were then normalized to give a final 'nonregrowth index' of the same order of magnitude than the \% membrane-ruptured cells below.

Inducing and revealing necrotic cell death. For induction of $N C D, 22,23$ exponentially growing HMX44A.atg1-1 vegetative cells were washed once and resuspended in 10 mM MES, pH 6.4, (MES hydrate, ref. M2933; Sigma; prepared in demineralized water) in Lab-Tek chambers as above. Cells were incubated for $16 \mathrm{~h}$ in MES and $3 \mathrm{mM}$ CAMP, then washed once in MES and incubated in either MES or MES with DIF-1 or derivatives thereof. After the indicated period of time, cells in the Lab-Tek chambers were examined by microscopy as above, and FACS determinations of DC-FDA fluorescence and cell membrane rupture were performed.

Possible production of ROS was assessed by microscopy and flow cytometry analysis. ${ }^{23}$ For each point of analysis, cells were incubated for $15 \mathrm{~min}$ at room temperature, protected from light, with DC-FDA $(10 \mu \mathrm{M}$; Sigma-Aldrich). For microscopy, cells in Lab-Tek culture chambers were examined directly after incubation by microscopy using Filter set 09 (Zeiss). For flow cytometry, data acquisition was performed directly after incubation, on a FACScan cytometer with CELLQuest software (Becton Dickinson) using FL1 filter. Data were analyzed using FlowJo software (Tree Star Inc.). Data are shown as \% DC-FDA fluorescent cells, which reflect initial mitochondrial uncoupling events in NCD.

Flow cytometry enables to quantify the proportion of cells with a disrupted membrane. Cells in Lab-Tek chambers were resuspended and transferred to tubes 
and data acquisition was performed on a FACScan cytometer with CELLQuest software (Becton Dickinson) for forward scatter (FSC) and side scatter (SSC) parameters that allowed to distinguish cells with a disrupted membrane from those with an intact one. ${ }^{23}$ To exclude small debris, a FSC threshold was applied based on the light properties of the cell populations in FSC and SSC modes. In brief, 5000 or 10000 events were collected per sample. Data analysis was carried out with CELLQuest software. Data are shown as \% membrane-ruptured cells, which reflects the end point of NCD.

Acknowledgements. We thank Professor Robert R Kay (MRC Laboratory of Molecular Biology, Cambridge, UK) for an essential suggestion leading to this work, and $\mathrm{E}$ Tresse, C Giusti and L Leserman for advice. This work was supported through institutional grants by INSERM and CNRS, and through specific grants by Agence Nationale pour la Recherche (DictyDeath ANR-05-BLAN-0333-01), the European Community (FP6 STREP TransDeath LSHG-CT-2004-511983), the Ministère pour la Recherche (ACl BCMS174), Cancéropôle PACA and Association pour la Recherche sur le Cancer.

1. Zong WX. Thompson CB. Necrotic death as a cell fate. Genes Dev 2006; 20: 1-15.

2. Festjens N, Vanden Berghe T, Vandenabeele P. Necrosis, a well-orchestrated form of cell demise: signalling cascades, important mediators and concomitant immune response. Biochim Bbiophys Acta 2006; 1757: 1371-1387.

3. Golstein P, Kroemer G. Cell death by necrosis: towards a molecular definition. Trends Biochem Sci 2007: 32: 37-43.

4. Gozuacik D, Kimchi A. Autophagy and cell death. Curr Top Dev Biol 2007; 78: 217-245.

5. Levine B, Kroemer G. Autophagy in the Pathogenesis of Disease. Cell 2008; 132: 27-42.

6. Kay RR. Cell differentiation in monolayers and the investigation of slime mold morphogens. Methods Cell Biol 1987; 28: 433-448.

7. Eichinger L, Pachebat JA, Glockner G, Rajandream MA, Sucgang R, Berriman M et al. The genome of the social amoeba Dictyostelium discoideum. Nature 2005; 435: 43-57.

8. Kessin RH. The secret lives of Dictyostelium. Methods Mol Biol 2006; 346: 3-14.

9. Kuspa A, Loomis WF. The Genome of Dictyostelium discoideum. Methods Mol Biol 2006 346: $15-30$

10. Roisin-Bouffay C, Luciani MF, Klein G, Levraud JP, Adam M, Golstein P. Developmental cell death in Dictyostelium does not require paracaspase. J Biol Chem 2004; 279 11489-11494.

11. Lam D, Levraud JP, Luciani MF, Golstein P. Autophagic or necrotic cell death in the absence of caspase and bcl-2 family members. Biochem Biophys Res Commun 2007; 363: 536-541.

12. Morris HR, Taylor GW, Masento MS, Jermyn KA, Kay RR. Chemical structure of the morphogen differentiation inducing factor from Dictyostelium discoideum. Nature 1987; 328: $811-814$

13. Thompson CRL, Kay RR. The role of DIF-1 signaling in Dictyostelium development. Mol Cell 2000; 6: 1509-1514.
14. Austin MB, Saito T, Bowman ME, Haydock S, Kato A, Moore BS et al. Biosynthesis of Dictyostelium discoideum differentiation-inducing factor by a hybrid type I fatty acid-type III polyketide synthase. Nat Chem Biol 2006; 2: 494-502.

15. Saito T, Kato A, Kay RR. DIF-1 induces the basal disc of the Dictyostelium fruiting body. Dev Biol 2008; 317: 444-453.

16. Cornillon S, Foa C, Davoust J, Buonavista N, Gross JD, Golstein P. Programmed cell death in Dictyostelium. J Cell Sci 1994; 107: 2691-2704.

17. Levraud J-P, Adam M, Luciani M-F, De Chastellier C, Blanton RL, Golstein P. Dictyostelium cell death : early emergence and demise of highly polarized paddle cells. J Cell Biol 2003 160: 1105-1114.

18. Lam D, Kosta A, Luciani MF, Golstein P. The IP3 Receptor Is Required to Signal Autophagic Cell Death. Mol Biol Cell 2008; 19: 691-700.

19. Zhukovskaya NV, Fukuzawa M, Yamada Y, Araki T, Williams JG. The Dictyostelium bZIP transcription factor DimB regulates prestalk-specific gene expression. Development 2006 133: 439-448.

20. Huang E, Blagg SL, Keller T, Katoh M, Shaulsky G, Thompson CR. bZIP transcription factor interactions regulate DIF responses in Dictyostelium. Development 2006; 133 449-458.

21. Thompson $\mathrm{CR}$, Fu Q, Buhay C, Kay RR, Shaulsky G. A bZIP/bRLZ transcription factor required for DIF signaling in Dictyostelium. Development 2004; 131: 513-523.

22. Kosta A, Roisin-Bouffay C, Luciani MF, Otto GP, Kessin RH, Golstein P. Autophagy gene disruption reveals a non-vacuolar cell death pathway in Dictyostelium. J Biol Chem 2004 279: 48404-48409.

23. Laporte C, Kosta A, Klein G, Aubry L, Lam D, Tresse E et al. A necrotic cell death model in a protist. Cell Death Differ 2007; 14: 266-274.

24. Gokan N, Kikuchi H, Nakamura K, Oshima Y, Hosaka K, Kubohara Y. Structural requirements of Dictyostelium differentiation-inducing factors for their stalk-cell-inducing activity in Dictyostelium cells and anti-proliferative activity in K562 human leukemic cells. Biochem Pharmacol 2005; 70: 676-685

25. Kay RR. The biosynthesis of differentiation-inducing factor, a chlorinated signal molecule regulating Dictyostelium development. J Biol Chem 1998; 273: 2669-2675.

26. Insall R, Nayler O, Kay RR. DIF-1 induces its own breakdown in Dictyostelium. EMBO J 1992; 11: 2849-2854

27. Shaulsky G, Loomis WF. Mitochondrial DNA replication but no nuclear DNA replication during development of Dictyostelium. Proc Natl Acad Sci USA 1995; 92: 5660-5663.

28. Hehlgans $\mathrm{T}$, Pfeffer $\mathrm{K}$. The intriguing biology of the tumour necrosis factor/tumour necrosis factor receptor superfamily: players, rules and the games. Immunology 2005; 115: 1-20.

29. Citri A, Yarden Y. EGF-ERBB signalling: towards the systems level. Nat rev 2006; 7 505-516.

30. Teng KK, Hempstead BL. Neurotrophins and their receptors: signaling trios in complex biological systems. Cell Mol Life Sci 2004; 61: 35-48.

31. Mehlen P, Furne C. Netrin-1: when a neuronal guidance cue turns out to be a regulator of tumorigenesis. Cell Mol Life Sci 2005; 62: 2599-2616.

32. Sussman M. Cultivation and synchronous morphogenesis of Dictyostelium unde controlled experimental conditions In: Spudich JA (ed). Methods in Cell Biology. Harcourt Brace Jovanovich: New York, 1987 pp. 9-29

33. Kopachik W, Oochata W, Dhokia B, Brookman JJ, Kay RR. Dictyostelium mutants lacking DIF, a putative morphogen. Cell 1983; 33: 397-403. 